Bull. Austral. Math. Soc.

VOL. 64 (2001) [149-156]

\title{
UNIQUE CONTINUATION FOR NON-NEGATIVE SOLUTIONS OF QUASILINEAR ELLIPTIC EQUATIONS
}

\author{
Pietro Zamboni
}

\section{Dedicated to Filippo Chiarenza}

The aim of this note is to prove the unique continuation property for non-negative solutions of the quasilinear elliptic equation

$$
\operatorname{div} A(x, u, \nabla u)=B(x, u, \nabla u) .
$$

We allow the coefficients to belong to a generalised Kato class.

\section{INTRODUCTION}

In his paper on Schrödinger semigroups [12] Simon formulated the following conjecture

Let $\Omega$ be a bounded subset of $\mathbb{R}^{n}$ and $V$ a function defined in $\Omega$ whose extension with zero values outside $\Omega$ belongs to the Stummel-Kato class $S\left(\mathbb{R}^{n}\right)$ (see Definition 2.2). Then the Schrödinger operator $H=-\Delta+V$ has the unique continuation property,

that is, if $u \in H^{1}(\Omega)$ is a solution of equation $H u=0$ which vanishes of infinite order at one point $x_{0} \in \Omega$ (see Definition 4.2), then $u$ must be identically zero in $\Omega$.

A positive answer to Simon's conjecture was given by Fabes, Garofalo and Lin in [5] for radial potentials $V$.

At the same time Chanillo and Sawyer in [1] proved the unique continuation property for solutions of the inequality $|\Delta u| \leqslant|V||u|$, assuming $V$ in the Morrey space $L^{r, n-2 r}\left(\mathbb{R}^{n}\right)$ with $r>(n-1) / 2$ (see Definition 2.1).

In this note, following an idea of Chiarenza and Garofalo (see [3]), we extend both the above results to the non-negative solutions of a quasilinear elliptic equation of the form

$$
\operatorname{div} A(x, u, \nabla u)=B(x, u, \nabla u) .
$$

Received 16th November, 2000

The author wish to express his gratitude to Professor Richard Wheeden and Professor Nicola Garofalo for some useful talks and suggestions.

Copyright Clearance Centre, Inc. Serial-fee code: 0004-9727/01 \$A2.00+0.00. 
Precisely we show that a non-negative solution $u, u \not \equiv 0$, of (1.1) cannot have a zero of infinite order, assuming that suitable powers of the coefficients of (1.1) belong to the Morrey space $L^{r, n-p r}\left(\mathbb{R}^{n}\right)$, with $r \in(1, n / p)$, or to the function space $\widetilde{M}_{p}\left(\mathbb{R}^{n}\right)$ (see Theorem 5.1). We denote by $\widetilde{M}_{p}\left(\mathbb{R}^{n}\right)$ a generalisation of the Stummel-Kato class (see and Remark 2.5).

We point out that a crucial role in the proof of the Theorem 5.1 is played by Fefferman's inequality

$$
\int_{\mathbb{R}^{n}}|u(x)|^{p}|V(x)| d x \leqslant c \int_{\mathbb{R}^{n}}|\nabla u(x)|^{p} d x \quad \forall u \in C_{0}^{\infty}\left(\mathbb{R}^{n}\right),
$$

where $c$ is a positive constant depending on some norm of $V$. In Section 3 we give a new proof of (1.2) assuming $V \in \widetilde{M}_{p}$.

\section{SOME FUNCTION SPACES}

We begin this section giving some definitions.

Definition 2.1: (Morrey spaces) Let $q \geqslant 1, \lambda \in(0, n)$. We say that $f \in$ $L_{\text {loc }}^{q}\left(\mathbb{R}^{n}\right)$ belongs to $L^{q, \lambda}\left(\mathbb{R}^{n}\right)$ if

$$
\sup _{\substack{x \in \mathbb{R}^{n} \\ \rho>0}} \frac{1}{\rho^{\lambda}} \int_{B(x, \rho)}|f(y)|^{q} d y \equiv\|f\|_{q, \lambda}^{q}<+\infty
$$

Here and in the following, we denote by $B(x, \rho)$ the ball centred at $x$ with radius $\rho$. Whenever $x$ is not relevant we shall write $B_{\rho}$.

Definition 2.2: (Stummel-Kato class) Let $f \in L_{\text {loc }}^{1}\left(\mathbb{R}^{n}\right)$. For any $r>0$ we set

$$
\eta(r) \equiv \sup _{x \in \mathbb{R}^{n}} \int_{B(x, r)} \frac{|f(y)|}{|x-y|^{n-2}} d y .
$$

We say that $f$ belongs to $S\left(\mathbb{R}^{n}\right)$ if

$$
\lim _{r \rightarrow 0} \eta(r)=0
$$

Definition 2.3: Let $f \in L_{\text {loc }}^{1}\left(\mathbb{R}^{n}\right)$. For $p \in(1, n)$ and $r>0$ we set

$$
\phi(r) \equiv \sup _{x \in \mathbb{R}^{n}}\left(\int_{|x-y|<r} \frac{1}{|x-y|^{n-1}}\left(\int_{|z-x|<r} \frac{|f(z)|}{|z-y|^{n-1}} d z\right)^{1 /(p-1)} d y\right)^{(p-1)} .
$$

We say that $f$ belongs to the function space $\widetilde{M}_{p}\left(\mathbb{R}^{n}\right)$ if

$$
\phi(r)<+\infty, \quad \forall r>0
$$


DEFinition 2.4: We say that $f \in L_{\text {loc }}^{1}\left(\mathbb{R}^{n}\right)$ belongs to the function space $M_{p}\left(\mathbb{R}^{n}\right)$ if

$$
\lim _{r \rightarrow 0} \phi(r)=0,
$$

where $\phi(r)$ is defined as in Definition 2.3.

Some comments are now in order.

REMARK 2.5. We have

$$
\begin{aligned}
\text { (i) } & M_{p}\left(\mathbb{R}^{n}\right) \subset \widetilde{M}_{p}\left(\mathbb{R}^{n}\right) ; \\
\text { (ii) } & M_{2}\left(\mathbb{R}^{n}\right) \equiv S\left(\mathbb{R}^{n}\right) .
\end{aligned}
$$

(i) is trivial. Concerning (ii), Fubini's theorem implies

$$
\begin{aligned}
\int_{|x-y|<r} \frac{1}{|x-y|^{n-1}}\left(\int_{|z-x|<r}\right. & \left.\frac{|f(z)|}{|z-y|^{n-1}} d z\right) d y \\
& =\int_{|z-x|<r}|f(z)| \int_{|x-y|<r} \frac{1}{|x-y|^{n-1}} \frac{1}{|z-y|^{n-1}} d y d z .
\end{aligned}
$$

Since

$$
\int_{|x-y|<r} \frac{1}{|x-y|^{n-1}} \frac{1}{|z-y|^{n-1}} d y \sim \frac{1}{|z-x|^{n-2}},
$$

we get the conclusion.

Therefore both the function spaces $M_{p}\left(\mathbb{R}^{n}\right)$ and $\widetilde{M}_{p}\left(\mathbb{R}^{n}\right)$ are generalisations of $S\left(\mathbb{R}^{n}\right)$.

\section{ON FefFerman's INEQUality}

In this section we recall some known results concerning Fefferman's inequality

$$
\int_{\mathbb{R}^{n}}|u(x)|^{p}|f(x)| d x \leqslant c \int_{\mathbb{R}^{n}}|\nabla u(x)|^{p} d x \quad \forall u \in C_{0}^{\infty}\left(\mathbb{R}^{n}\right)
$$

and give a new proof assuming $f \in \widetilde{M}_{p}\left(\mathbb{R}^{n}\right)$.

In [7] Fefferman proved (3.1), in the case $p=2$, assuming $f \in L^{r, n-2 r}\left(\mathbb{R}^{n}\right)$, with $1<r \leqslant n / 2$.

Later in [10] Schechter showed the same result taking $f$ in the Stummel-Kato class $S\left(\mathbb{R}^{n}\right)$.

We stress that it is not possible to compare the assumptions $f \in L^{r, n-2 r}\left(\mathbb{R}^{n}\right)$ and $f \in S\left(\mathbb{R}^{n}\right)$.

Chiarenza and Frasca [2] generalised Fefferman's result proving (3.1) under the assumption $V \in L^{r, n-p r}\left(\mathbb{R}^{n}\right)$ with $r \in(1, n / p)$ and $p \in(1, n)$. Namely they proved the following 
152

P. Zamboni

[4]

Theorem 3.1. (See [2, p.407].) Assume $1<p<n, 1<r \leqslant n / p, f \in$ $L^{r, n-p r}\left(\mathbb{R}^{n}\right)$. Then there exists a constant $c$ depending on $n$ and $p$ such that

$$
\int_{\mathbb{R}^{n}}\left|u^{p}(x)\right||f(x)| d x \leqslant c\|f\|_{r, n-p r} \int_{\mathbb{R}^{n}}|\nabla u(x)|^{p} d x, \quad \forall u \in C_{0}^{\infty}\left(\mathbb{R}^{n}\right) .
$$

In the following theorem we provide a generalisation of Schecter's result, proving (3.1) under the assumption $f \in \widetilde{M}_{p}\left(\mathbb{R}^{n}\right), p \in(1, n)$.

TheOREM 3.2. Assume $f \in \widetilde{M}_{p}\left(\mathbb{R}^{n}\right)$. Then for any $r>0$ there exists a positive constant $c(n, p)$ such that

$$
\int_{\mathbb{R}^{n}}|f(x)||u(x)|^{p} d x \leqslant c(n, p) \phi(2 r) \int_{\mathbb{R}^{n}}|\nabla u(x)|^{p} d x
$$

for any $u \in C_{0}^{\infty}\left(\mathbb{R}^{n}\right)$ supported in $B\left(x_{0}, r\right)$.

Proof: For any $u \in C_{0}^{\infty}\left(\mathbb{R}^{n}\right)$ supported in $B\left(x_{0}, r\right)$, using the well known inequality

$$
|u(x)| \leqslant c(n, p) \int_{B\left(x_{0}, r\right)} \frac{|\nabla u(y)|}{|x-y|^{n-1}} d y
$$

and Fubini's theorem, we have

$$
\begin{aligned}
\int_{\mathbb{R}^{n}} \mid & \left.f(x)|| u(x)\right|^{p} d x \\
& =\int_{B\left(x_{0}, r\right)}|f(x)||u(x)|^{p} d x \\
& \leqslant c(n, p) \int_{B\left(x_{0}, r\right)}|f(x)||u(x)|^{p-1}\left(\int_{B\left(x_{0}, r\right)} \frac{|\nabla u(y)|}{|x-y|^{n-1}} d y\right) d x \\
\leqslant & c(n, p) \int_{B\left(x_{0}, r\right)}|\nabla u(y)|\left(\int_{B\left(x_{0}, r\right)}|f(x)||u(x)|^{p-1} \frac{1}{|x-y|^{n-1}} d x\right) d y \\
\leqslant & c(n, p)\left(\int_{B\left(x_{0}, r\right)}|\nabla u(y)|^{p} d y\right)^{1 / p} \cdot \\
& \cdot\left[\int_{B\left(x_{0}, r\right)}\left(\int_{B\left(x_{0}, r\right)}|f(x)||u(x)|^{p-1} \frac{1}{|x-y|^{n-1}} d x\right)^{p /(p-1)} d y\right]^{(p-1) / p} .
\end{aligned}
$$

https://doi.org/10.1017/S0004972700019766 Published online by Cambridge University Press 
We also have

$$
\begin{aligned}
\int_{B\left(x_{0}, r\right)} & \left(\int_{B\left(x_{0}, r\right)}|f(x)||u(x)|^{p-1} \frac{1}{|x-y|^{n-1}} d x\right)^{p /(p-1)} d y \\
& \leqslant \int_{B\left(x_{0}, r\right)}\left(\int_{B\left(x_{0}, r\right)} \frac{|f(z)|}{|z-y|^{n-1}} d x\right)^{1 /(p-1)} \int_{B\left(x_{0}, r\right)} \frac{|f(x)||u(x)|^{p}}{|x-y|^{n-1}} d x d y \\
& =\int_{B\left(x_{0}, r\right)}|f(x)||u(x)|^{p} \int_{B\left(x_{0}, r\right)} \frac{1}{|x-y|^{n-1}}\left(\int_{B\left(x_{0}, r\right)} \frac{|f(z)|}{|z-y|^{n-1}} d z\right)^{1 /(p-1)} d y d x \\
& \leqslant \phi^{1 /(p-1)}(2 r) \int_{B\left(x_{0}, r\right)}|f(x)||u(x)|^{p} d x .
\end{aligned}
$$

By (3.3) and (3.4) we obtain the desired conclusion.

REMARK 3.3. We note that proceeding as in Theorem 3.2 using the representation formula (see, for example [6])

$$
\left|u(x)-u_{B_{R}}\right| \leqslant c \int_{B_{R}} \frac{|\nabla u(y)|}{|x-y|^{n-1}} d y,
$$

instead of (3.2), it is possible to obtain a Poincaré type inequality. Namely

THEOREM 3.4. Suppose $u$ is a Lipschitz continuous function on $\bar{B}_{R}$, the closure of $B_{R}$, and $f$ is a function defined on $B_{R}$ whose extension with zero values outside $B_{R}$ belongs to $\widetilde{M}_{p}\left(\mathbb{R}^{n}\right)$. Then there exists a positive constant $c$ such that

$$
\int_{B_{R}}|f(x)|\left|u(x)-u_{B_{R}}\right|^{p} d x \leqslant c \phi(2 R) \int_{B_{R}}|\nabla u(x)|^{p} d x
$$

where $u_{B_{R}}$ is the average $\left(1 /\left|B_{R}\right|\right) \int_{B_{R}} u(x) d x$ where $\left|B_{R}\right|$ is the Lebesgue measure of $B_{R}$.

\section{ASSUMPTIONS AND PRELIMINARY RESULTS}

Let $\Omega$ be a bounded open set in $\mathbb{R}^{n}$. The equation we consider is of the form

$$
\operatorname{div} A(x, u, \nabla u)=B(x, u, \nabla u)
$$

where

$$
A(x, u, \xi): \Omega \times \mathbb{R} \times \mathbb{R}^{n} \rightarrow \mathbb{R}^{n}
$$

and

$$
B(x, u, \xi): \Omega \times \mathbb{R} \times \mathbb{R}^{n} \rightarrow \mathbb{R}
$$


are two continuous functions satisfying the following conditions

$$
\left\{\begin{array}{l}
|A(x, u, \xi)| \leqslant a|\xi|^{p-1}+b(x)|u|^{p-1} \\
|B(x, u, \xi)| \leqslant c(x)|\xi|^{p-1}+d(x)|u|^{p-1} \\
\xi A(x, u, \xi) \geqslant|\xi|^{p}-d(x)|u|^{p}
\end{array}\right.
$$

for almost all, $x \in \Omega, \forall u \in \mathbb{R}, \forall \xi \in \mathbb{R}^{n}$. We assume that $p$ is a fixed number in $(1, n)$, $a$ is a positive constant and $b, c$ and $d$ are measurable functions in $\Omega$ whose extension with zero values outside $\Omega$ are such that

$$
b^{p /(p-1)}, c^{p}, d \in M_{p}\left(\mathbb{R}^{n}\right),
$$

or

$$
b^{p /(p-1)}, c^{p}, d \in L^{r, n-p r}\left(\mathbb{R}^{n}\right) \quad r \in(1, n / p) .
$$

DEFINITION 4.1: We say that a function $u \in H_{\mathrm{loc}}^{1, p}(\Omega)$ is a local weak solution of (4.1) in $\Omega$ if

$$
\int_{\Omega}\{A(x, u(x), \nabla u(x)) \nabla \phi(x)+B(x, u(x), \nabla u(x)) \phi(x)\} d x=0
$$

for every $\phi \in C_{0}^{\infty}(\Omega)$.

We remark that Definition 4.1 is meaningful by Theorem 3.1 or Theorem 3.2.

To state our result we need one more definition.

DEFINITION 4.2. Assume $w \in L_{\text {loc }}^{1}(\Omega), w \geqslant 0$ almost everywhere in $\Omega$. We say that $w$ has a zero of infinite order at $x_{0} \in \Omega$ if

$$
\lim _{\sigma \rightarrow 0} \frac{\int_{B\left(x_{0}, \sigma\right)} w(x) d x}{\left|B\left(x_{0}, \sigma\right)\right|^{k}}=0 \quad \forall k>0 .
$$

The following two lemmas are known.

Lemma 4.3. (See [9].) Assume $w \in L_{\text {loc }}^{1}(\Omega), w \geqslant 0$ almost everywhere in $\Omega$, $w \not \equiv 0$. If

$$
\exists C>0: \int_{B\left(x_{0}, 2 \sigma\right)} w(x) d x \leqslant C \int_{B\left(x_{0}, \sigma\right)} w(x) d x \quad \forall \sigma>0,
$$

then $w(x)$ has no zero of infinite order in $\Omega$.

LEMMA 4.4. (See [4] and [8].) Let $B_{\tilde{r}} \subset \mathbb{R}^{n}, u \in H^{1, p}\left(B_{\tilde{r}}\right)$ be and assume that for all $B_{r} \subset B_{\tilde{r}}$ there exists a constant $K$ such that

$$
\left(\int_{B_{r}}|\nabla u(x)|^{p} d x\right)^{1 / p} \leqslant K r^{(n-p) / p}
$$

Then there exist two positive constants $\delta$ and $C$, depending on $K, p, n$, such that

$$
\left(\int_{B_{\tilde{r}}} e^{\delta u(x)} d x\right)\left(\int_{B_{\tilde{r}}} e^{-\delta u(x)} d x\right) \leqslant C\left|B_{\tilde{r}}\right|^{2} .
$$




\section{UNIQUE CONTINUATION}

In this section we state and prove our result, namely

THEOREM 5.1. Let $u \in H^{1}(\Omega), u \geqslant 0, u \not \equiv 0$, be a solution of (4.1) satisfying (4.2) and (4.3) or (4.2) and (4.3)'.

Then $u$ has no zero of infinite order in $\Omega$.

Proof: Let $x_{0} \in \Omega$, let $B\left(x_{0}, R\right)$ be a ball such that $B\left(x_{0}, 2 R\right)$ is contained in $\Omega$. Consider any $B_{h}$ contained in $B\left(x_{0}, R\right)$. Let $\eta$ be a non negative smooth function with support in $B_{2 h}$. Using $\phi=\eta^{p} u^{1-p}$ as test function in (4.4) we get (see [11])

$$
\int_{\Omega}|\nabla \log u(x)|^{p} \eta^{p}(x) d x \leqslant C_{1}(p, a)\left\{\int_{\Omega}|\nabla \eta(x)|^{p} d x+\int_{\Omega} V(x) \eta^{p}(x) d x\right\}
$$

where $V$ is defined by

$$
V=b^{p /(p-1)}+c^{p}+d
$$

By Theorem 3.1 or Theorem 3.2, we have

$$
\int_{\Omega} V(x) \eta^{p}(x) d x \leqslant C_{2}(\operatorname{spt} \eta) \int_{\Omega}|\nabla \eta(x)|^{p} d x
$$

Inserting this inequality in (5.1), we obtain

$$
\int_{\Omega} \eta^{p}(x)|\nabla \log u(x)|^{p} d x \leqslant C_{3}(p, a, \operatorname{diam} \Omega) \int_{\Omega}|\nabla \eta(x)|^{p} d x
$$

Choosing $\eta$ so that $\eta=1$ in $B_{h}$ and $|\nabla \eta| \leqslant 3 / h$, by (5.2) we have

$$
\int_{B_{h}}|\nabla \log u(x)|^{p} d x \leqslant C_{4}(p, a, \operatorname{diam} \Omega) h^{n-p}
$$

Therefore, by Lemma 4.4, we have

$$
\int_{B_{h}} u^{\delta}(x) d x \int_{B_{h}} u^{-\delta}(x) d x \leqslant C\left|B_{h}\right|^{2}
$$

that is, $u^{\delta}$ belongs to the Muckenhoupt class $A_{2}$ for some $\delta>0$ (see [3] and [6]). Now it is well known that $A_{2}$ implies the doubling property for $u_{\delta}$, that is, the assumption of Lemma 4.3. So the conclusion follows for $u^{\delta}$ and hence also for $u$. 


\section{REFERENCES}

[1] S. Chanillo and A.E. Sawyer, 'Unique continuation for $\Delta+v$ and the C. Fefferman Phong class', Trans. Amer. Math. Soc. 318 (1990), 275-300.

[2] F. Chiarenza and M. Frasca, 'A remark on a paper by C. Fefferman', Proc. Amer. Math. Soc. 108 (1990), 407-409.

[3] F. Chiarenza and N. Garofalo, Unique continuation for non-negative solutions of Schrödinger operators, (Institute for Mathematics and its Applications, Preprint Series No. 122) (University of Minnesota, 1984).

[4] R.R. Coifmann and C. Fefferman, 'Weighted norm inequalities for maximal functions and singular integrals', Studia Math. 51 (1974), 241-250.

[5] E. Fabes, N. Garofalo and F.H. Lin, 'A partial answer to a conjecture of B. Simon concerning unique continuation', J. Funct. Anal. 88 (1990), 194-210.

[6] E. Fabes, C. Kenig and R. Serapioni, 'The local regularity of solutions of degenerate elliptic equations', Comm. Partial Differential Equations 7 (1982), 77-116.

[7] C. Fefferman, 'The uncertainty principle', Bull. Amer. Math. Soc. 9 (1983), 129-206.

[8] J. Garcia Cuerva and J.L. Rubio de Francia, Weighted norm inequalities and related topics (North-Holland, Amsterdam, 1985).

[9] M. Giaquinta, Multiple integrals in the calculus of variation and linear and nonlinear elliptic systems, Annals of Math. Studies 105 (Princeton University Press, Princeton, NJ, 1983).

[10] M. Schechter, Spectra of partial differential operators (second edition), Applied Maths and Mechanics 14 (North Holland Publishing Co., Amsterdam, New York, 1986).

[11] J. Serrin, 'Local behavior of solutions of quasilinear equations', Acta Math. 111 (1964), 247-302.

[12] B. Simon, 'Schrödinger semigroups', Bull. Amer. Math. Soc. 7 (1982), 447-526.

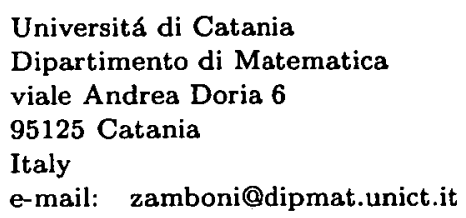

